\title{
Key Elements of Mammography Shared Decision-Making: a Scoping Review of the Literature
}

\author{
Lori L. DuBenske, $P h D^{7}$, Sarina B. Schrager, $M D^{7}$, Mary E. Hitchcock, MLS², Amanda K. Kane, BS , \\ Terry A. Little, $M S^{l}$, Helene E. McDowell, $M S^{3}$, and Elizabeth S. Burnside, $M D^{7}$
}

${ }^{1}$ School of Medicine and Public Health, University of Wisconsin-Madison, Madison, WI, USA; ${ }^{2}$ Ebling Library for the Health Sciences, University of Wisconsin-Madison, Madison, WI, USA; ${ }^{3}$ Health Decision Support, Inc., Madison, WI, USA.

BACKGROUND: New guidelines recommend shared decision-making (SDM) for women and their clinician in consideration of breast cancer screening, particularly for women ages 35-50 where guidelines for routine mammography are controversial. A number of models offer general guidelines for SDM across clinical practice, yet they do not offer specific guidance about conducting $\mathrm{SDM}$ in mammography. We conducted a scoping review of the literature to identify the key elements of breast cancer screening SDM and synthesize these key elements for utilization by primary care clinicians.

METHODS: The Cochrane Database of Systematic Reviews; Cumulative Index to Nursing and Allied Health Literature (CINAHL Plus); PsycInfo, PubMed (MEDLINE), Scopus, and SocIndex databases were searched. Inclusion criteria were original studies from peer-reviewed publications (from 2009 or later) reporting breast cancer screening (mammography), medical decision-making, and patient-centered care. Study populations needed to include female patients 18+ years of age facing a real-life breast cancer screening decision. Article findings were specific to shared decision-making and/or use of a decision aid. Data extracted includes study design, population, setting, intervention, and critical findings related to breast cancer screening SDM elements. Scoping analysis includes descriptive analysis of study features and content analysis to identify the SDM key elements.

RESULTS: Twenty-four articles were retained. Three thematic categories of key elements emerged from the extracted elements: information delivery/patient education (specific content and delivery modes), interpersonal clinician-patient communication (aspects of interpersonal relationship impacting SDM), and framework of the decision (sociocultural factors beyond direct SDM deliberation). A number of specific breast cancer screening SDM elements relevant to primary care clinical practice are delineated.

DISCUSSION: The findings underscore the importance of the relationship between the patient and clinician and the necessity of spelling out each step in the SDM process. The clinician needs to be explicit in telling a woman that she has a choice about whether to get a mammogram and the benefits and harms of screening mammography. Finally, clinicians need to be aware of sociocultural factors

Received March 8, 2018

Revised May 29, 2018

Accepted July 3, 2018

Published online July 20, 2018 that can influence their relationships and their patients' decision-making processes and attempt to identify and address these factors.

KEY WORDS: mammography; shared decision-making; breast cancer screening; patient-centered care; primary care; women's health; health communication.

J Gen Intern Med 33(10): 1805-14

DOI: $10.1007 / \mathrm{s} 11606-018-4576-6$

(C) Society of General Internal Medicine 2018

\section{BACKGROUND}

Breast cancer is the leading cause of death for women 3549 years old. ${ }^{1,2}$ Screening mammography plays an important public health role in reducing breast cancer mortality and contributes substantially to the national and statewide declines in breast cancer mortality over the past 30 years. ${ }^{2}$ However, the benefits-to-harms ratio associated with mammography is less favorable in younger women as compared to higher incidence age ranges. Harms like anxiety, false positives, and overdiagnosis make shared decision-making (SDM) an important component of screening programs. Optimal utilization of mammography is one key to assuring health and well-being of women and improved public health outcomes. Yet mammography for women ages 40-49 remains controversial and confusing for patients. The United States Preventive Services Task Force (USPSTF) recommends personalized decision-making for average risk women ages $40-49 .^{3}$ The American Cancer Society (ACS) recently published guidelines that recommend annual screening from ages 45 to $54 .{ }^{4} \mathrm{New}$ guidelines from American College of Obstetrics Gynecology (ACOG) also recommend SDM for women under age 50. 5,6 These variations are largely due to organizations assigning different merit to evidence from clinical trials, observational studies, and the balance of benefits and harms for different groups of women. $^{3,4,7}$

With the goal of an informed and joint decision, SDM, most simply defined, is "a process in which patients are involved as active partners with the clinician in clarifying acceptable medical options and in choosing a preferred course of clinical care." 8 Although the literature on effective shared decisionmaking is robust, little research focuses on what elements are 
necessary for implementing SDM as it relates to breast cancer screening.

A number of models exist for general shared decisionmaking, such as the Agency for Healthcare Research and Quality's SHARE method, ${ }^{9}$ the 5 As method described by the USPSTF, ${ }^{8}$ the "IAIS" model ${ }^{10}$, and the 3-step "talk" model. ${ }^{11}$ Each of these models outlines elements or steps of the decision-making process. Although these models offer general guidelines for SDM across clinical practice, they do not offer specific guidance about conducting SDM for breast cancer screening.

Our recent study examining clinicians' and women's experiences of breast cancer screening decision-making revealed that women and clinicians agreed about the value of SDM; however, discrepancies exist in their expectations and goals. ${ }^{12}$ Women generally trust that their clinicians will counsel them based on knowledge of breast cancer risk factors and screening guidelines. On the other hand, clinicians reported a lack of confidence and knowledge regarding individual breast cancer risk assessment and time constraints as barriers to SDM. These initial findings emphasize the need for specific and effective guidance in the breast cancer screening SDM.

We conducted a scoping review of the literature to identify the key elements of breast cancer screening SDM and synthesized these key elements for utilization by primary care clinicians. This review is part of a broader study to inform the development and evaluation of a breast cancer screening SDM aid for primary care clinicians and their patients.

\section{METHODS}

Although not a systematic review, this scoping review conforms to the Preferred Reporting Items for Systematic Reviews and Meta-Analyses (PRISMA) standards. ${ }^{13}$ A scoping review is defined as "a form of knowledge synthesis that addressed an exploratory research question aimed at mapping key concepts, types of evidence, and gaps in research related to a defined area or field by systematically searching, selecting, and synthesizing existing knowledge."14 The nature of the literature did not facilitate a systematic review or metaanalysis, due to the heterogeneous representation of methodologies, limited number of interventions, and varied outcomes reported.

\section{Database Search}

The following databases were searched on Nov 11, 2016: Cochrane Database of Systematic Reviews; Cumulative Index to Nursing and Allied Health Literature (CINAHL Plus); PsycInfo, PubMed (MEDLINE), Scopus, and SocIndex. Additional articles found via hand searching references or similar articles in PubMed were also reviewed. On December 12, 2016, a secondary search was conducted in the previously mentioned databases to identify items missed in the first round of searching.

Search terms are presented in Table 1. After an initial general search, a secondary search was conducted to increase specificity of the items located.

Inclusion criteria were original studies or editorials addressing empirical evidence from peer reviewed publications (from 2009 or later) reporting breast cancer screening (mammography), medical decision-making, and patient-centered care (represented in Fig. 1). Study populations needed to include female patients $18+$ years of age facing a real-life mammography decision (not a hypothetical scenario) on their own behalf. Article findings were specific to shared decisionmaking and/or use of a patient decision aid.

Exclusion criteria were studies prior to 2009; editorial articles absent of cited empirical evidence (e.g., opinion pieces or reflections on policy); articles discussing expectations, but not providing patient-reported data; protocols; abstracts; and articles not written in English. We also excluded case studies given lack of generalizability of small sample size.

\section{Article Selection}

The lead author (LD) and one collaborator (AK) initially screened titles and abstracts identified by the search for inclusion or exclusion criteria. Any instances of discordance between reviewers were resolved through mutual discussion and agreement. Studies that remained uncertain due to limited detail in the abstract were pulled for full article review.

For those titles/abstracts that were identified as potentially meeting the inclusion criteria, the full articles were obtained

Table 1 Literature Review Search Terms

Initial general search terms

Secondary search terms to increase specificity

(screen* OR mammogram*) AND (“patient preferences" OR “clinician-patient communication" OR discussion
OR communicat* OR “decision mak*" OR support)
(((((screening) AND Decision[tw] OR choice[tiab]) AND (support[tw] OR aid[tw] OR make[tw] OR
making[tiab]) OR "patient preference"[tw] OR "patient preferences"[tw]) AND (((Patient[tw] OR woman[tiab]
OR women[tiab] OR female[tiab]) AND (doctor[tiab] OR clinician[tiab] OR clinician[tiab] OR clinician[tw]))
AND communication[tw] OR communicate[tw] OR discuss[tiab] OR discussion[tiab])) AND (("breast"[tw]
AND (cancer[tw] OR neoplasms[tw] OR screen[tiab] OR screening[tw])) OR mammogram[tw] OR
mammography[tw]))) AND ((((Decision[tw] OR choice[tiab]) AND (support[tw] OR aid[tw] OR make[tw] OR
making[tiab]) OR "patient preference"[tw] OR "patient preferences"[tw]) AND (((Patient[tw] OR woman[tiab]
OR women[tiab] OR female[tiab]) AND (doctor[tiab] OR clinician[tiab] OR clinician[tiab] OR clinician[tw]))
AND communication[tw] OR communicate[tw] OR discuss[tiab] OR discussion[tiab])) AND (("breast"[tw]
AND (cancer[tw] OR neoplasms[tw] OR screen[tiab] OR screening[tw])) OR mammogram[tw] OR
mammography[tw] AND screening[tw]))




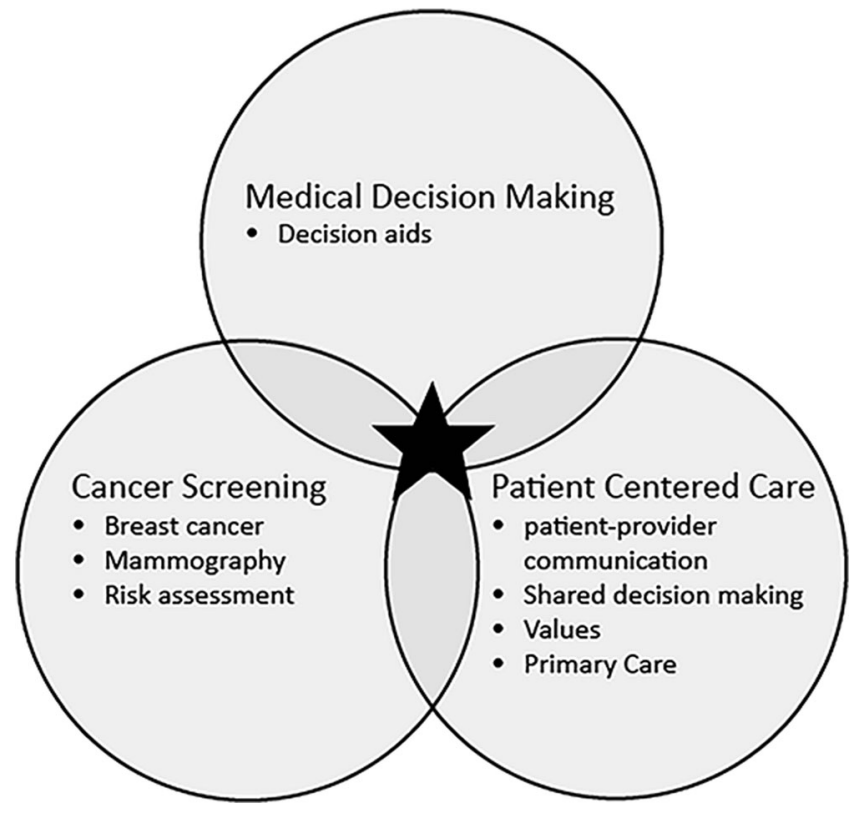

Figure 1 Schema for literature search topics.

and were subject to further review. The lead author (LD) and one co-collaborator (AK) independently selected articles based on the inclusion and exclusion criteria. Discrepancies in article selection were resolved through discussion between reviewers. Inclusion/exclusion decisions were recorded.

\section{Data Extraction}

Data extracted from each reviewed article includes the following elements: study design, population, setting, intervention (if any), and critical findings related to breast cancer screening SDM elements (e.g., structural components, processes, and delivery modes). Data extracted from systematic review articles was cross-checked against other included articles to prevent duplication of evidence. The lead author (LD) performed data extraction for each of the included articles. To increase reliability, three collaborators (SS, EB, and EJ) also performed data extraction on equal sized subsets of included articles. Any issues of discrepancy in extracting SDM elements were resolved through mutual discussion.

\section{Scoping Analysis}

Scoping analysis includes (1) descriptive analysis of features of the studies (e.g., population, design) and (2) content analysis to identify the SDM key elements. To facilitate the synthesis of a broad nature of SDM elements, content analysis included the development of a thematic categorization schema based on the nature of issues addressed by the extracted SDM elements. Articles may have findings that addressed more than one thematic category and as such, these articles are crosscoded. The collaborators discussed the content of the scoping analysis. The lead author (LD) wrote the scoping analysis, which was then checked independently by three collaborators
(SS, EB, and HM) who fed back their comments. Any disagreements were discussed and resolved.

\section{RESULTS}

The PRISMA flow diagram (Fig. 2) summarizes the literature review. Six hundred ninety-three unique records were identified. Sixteen articles were excluded because they were not written in English. Twenty-four articles were found to meet inclusion criteria for analysis.

\section{Descriptive Analysis}

Table 2 provides a summary of included articles. Studies are primarily from the USA $(n=18)$. The studies included the following subject populations: women engaged in mammography screening only $(n=12)$; heterogeneous patient population engaged in heterogeneous medical screening including mammography $(n=8)$; clinicians/experts only $(n=2)$; mixed women and clinicians $(n=2)$.

Heterogeneous methodologies included six qualitative studies, 12 quantitative studies (including six cross-sectional surveys, two pre-posttest, three RCT, and one implementation-effectiveness trial), one mixed methods design, three systematic reviews, and two editorials that included original data. Nine studies included development or evaluation of a decision aid tool.

\section{Content Analysis}

Three thematic categories of key elements emerged from the extracted elements: information delivery/patient education (ID), interpersonal clinician-patient communication (COM), and framework of the decision (FD). Findings within these categories are summarized below. While a number of SDM elements were identified and listed in Table 2, Box 1 provides an overview highlighting elements that are most applicable to clinical practice.

\section{Box 1 Key elements for the clinician}

Information delivery and patient education

- Discuss the benefits AND risks of mammography

- When conveying numbers, use absolute risk reduction and natural frequencies

- Use visual aids to increase comprehension

- Use descriptive everyday language rather than technical terms (e.g., "normal test result" rather than "negative")

- Use neutral and balanced tone and active language (e.g., "you" rather than "one")

- Use real-world examples

Interpersonal clinician-patient communication

- Assess the woman's understanding of the information

- Explore the woman's personal preferences

- Consider multiple cultural perspectives and language barriers

- Express enthusiasm and encouragement for decision-making Framework of the decision

- Underscore that it is a woman's choice to have a mammogram or not

- Recognize that there are multiple factors beyond the SDM communication that can impact a woman's process of making a decision (i.e., race, ethnicity, trust of medical system, perceived risk, self-efficacy)

- Be aware that personal biases may affect SDM 


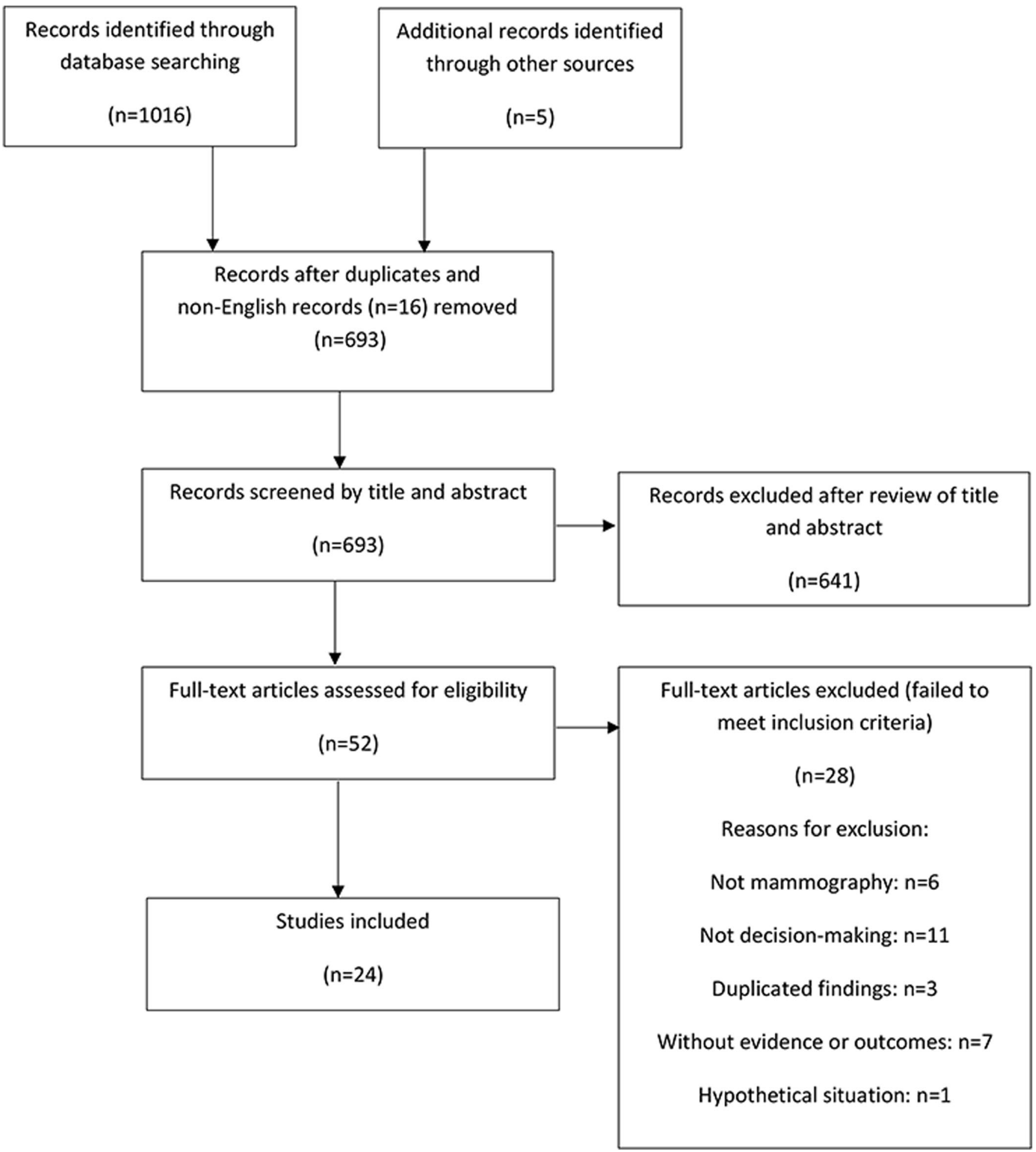

Figure 2 Scoping review process using an adaptation of the Preferred Reporting Items for Systematic Reviews and Meta-analysis (PRISMA) schema.

Information Delivery and Patient Education. ID addresses the nature of the content provided to the patient or clinician, as well as the format for delivery. Methods of delivery include direct clinician-patient communication or from an educational tool, such as a printed or online decision aid. Nineteen of the articles addressed a key element in this category.
Models of SDM highlight the importance of discussing alternatives along with the benefits and risks of each alternative, as well as uncertainties. ${ }^{18,21,29}$ However, in a national survey of patients who received breast, prostate, or colorectal cancer screening, those who received breast cancer screening were least likely to report their clinician discussed benefits or risks of screening. ${ }^{22,24}$ Furthermore, discussion of risks is 
Table 2 List of Included Articles with Brief Description of Methodology and SDM Elements Findings

\begin{tabular}{ll}
\hline \hline $\begin{array}{l}\text { First author/year/ } \\
\text { country }\end{array}$ & $\begin{array}{l}\text { a. Subjects } \\
\text { b. Setting } \\
\text { c. Methodology } \\
\text { d. Intervention (if applicable) }\end{array}$ \\
\hline Uhmed $^{15}$ & $\begin{array}{l}\text { a. Women/mammogram } \\
\text { b. Clinical practice } \\
\text { c. Review }\end{array}$ \\
Allen 2013 USA $^{16}$ & $\begin{array}{l}\text { a. Women/mammogram (40-50 y; Caucasian, } \\
\text { Latina, African American) } \\
\text { b. Community } \\
\text { c. Qualitative: focus groups }\end{array}$ \\
&
\end{tabular}

\begin{tabular}{|c|c|}
\hline \multirow{3}{*}{$\begin{array}{l}\text { Barrett } 2011 \\
\text { USA }^{17}\end{array}$} & a. Heterogeneous screening \\
\hline & b. Multiple settings \\
\hline & c. Review \\
\hline \multirow{3}{*}{$\mathrm{USA}^{18}$} & a. Women/mammogram \\
\hline & b. Clinical practice \\
\hline & c. Editorial \\
\hline \multirow{2}{*}{$\begin{array}{l}\text { Dillard } 2010 \\
\text { USA }^{19}\end{array}$} & a. Heterogeneous screening \\
\hline & $\begin{array}{l}\text { b. Nationwide telephone survey } \\
\text { c. Cross-sectional survey }\end{array}$ \\
\hline \multirow{2}{*}{$\begin{array}{l}\text { Edwards } 2013 \\
\mathrm{UK}^{20}\end{array}$} & a. Heterogeneous screening \\
\hline & b. Multiple settings \\
\hline \multirow{3}{*}{ Forbes $2014 \mathrm{UK}^{21}$} & c. Systematic review \\
\hline & $\begin{array}{l}\text { a. Women/mammogram and experts } \\
\text { b. Community sample }\end{array}$ \\
\hline & c. Brochure development feedback with interviews \\
\hline \multirow{3}{*}{$\begin{array}{l}\text { Fowler } 2013 \\
\text { USA }^{22}\end{array}$} & a. Heterogeneous screening \\
\hline & b. National sample \\
\hline & c. Cross-sectional survey \\
\hline \multirow[t]{3}{*}{ Gunn 2015 USA $^{23}$} & a. Women/mammogram $(\leq 50 \mathrm{y})$ \\
\hline & b. National sample (HINTS) \\
\hline & c. Cross-sectional survey \\
\hline \multirow{3}{*}{$\begin{array}{l}\text { Hoffman } 2014 \\
\text { USA }^{24}\end{array}$} & a. Heterogeneous screening $(50+y)$ \\
\hline & b. National sample \\
\hline & c. Cross-sectional survey \\
\hline \multirow[t]{3}{*}{ Klein 2016 USA $^{25}$} & a. Women/mammogram $(40-49$ y) \\
\hline & b. Clinic setting \\
\hline & $\begin{array}{l}\text { c. Mixed method: pre-post and qualitative } \\
\text { d. Mammopad tool }\end{array}$ \\
\hline \multirow{4}{*}{$\begin{array}{l}\text { Mathiew } 2010 \\
\text { AUS }^{26}\end{array}$} & a. Women/mammogram (38-45 y) \\
\hline & b. Community sample \\
\hline & $\begin{array}{l}\text { c. RCT of online mamm vs delayed access control } \\
\text { group }\end{array}$ \\
\hline & d. Online mammogram decision aid \\
\hline \multirow{3}{*}{$\begin{array}{l}\text { Nekhlyudov } 2009 \\
\text { USA }^{29}\end{array}$} & a. Clinicians \\
\hline & b. Academic and clinical setting \\
\hline & $\begin{array}{l}\text { c. Pilot test with qualitative feedback } \\
\text { d. Communication manual for clinicians }\end{array}$ \\
\hline
\end{tabular}

$\begin{array}{ll}\text { Pasternack 2011 } & \text { a. Women/mammogram and clinicians } \\ \text { FIN }^{30} & \text { b. Clinic setting } \\ & \text { c. Pilot test w/ qualitative feedback } \\ & \text { d. Decision Aid leaflet w/ invitation letter } \\ \text { Peterson } 2016 & \text { a. Heterogeneous screening } \\ \text { USA }^{31} & \text { b. Multiple settings } \\ & \text { c. Systematic review }\end{array}$

\section{SDM elements [category designation]}

Risk communication is 2-way exchange of information and opinion. The format of presentation is important: ARR more balanced and understandable representation of risk for patients and doctors; natural frequency easier to understand and interpret than $\%$ or probability; support for personalized risk communication [ID]

Few women felt confident taking active role in DM [FD]; African American theme of need to be prepared for discussion with doctor [FD]; Latina more likely defer to clinician recommendation [FD]; skepticism of information regarding disadvantage of mammogram [FD]; others thought information had merit but concern for own ability to sift through inconsistent information and go against doctor recommendation [FD]. Conclusion: difficult to predict whether greater involvement with clinician [COM] would necessarily influence appropriate use of mammogram without significant trust-building efforts, as notion of weighing benefit to harms seemed irrelevant to many women [FD]

Natural frequency presentation offers clear and coherent means of presenting statistical information and can be advocated and incorporated into evidence-based and PT-oriented clinical practice [ID]

Argue to disclose risk of overdiagnosis [ID]

Perceived increased risk of cancer [FD] associated with increased information seeking alone and with clinician [COM], but preference for decision-making involvement was consistent across perceived risk levels [FD]

Personalized risk estimates incorporated within communication interventions for screening programs enhanced informed choice [ID]

Offers women's preferences for presentation of specific mamm information in informational brochure in UK [ID]

Among cancer screening, mammogram PTs least likely believe they had a choice [FD], least likely discuss pros of screen [ID], least likely for clinicians to illicit PT input in DM [COM]; almost no discussions of cons of screening [ID]

Mammogram positive correlated with perceived choice for mammogram [FD]

Most discussions addressed pros of screening [ID]; few addressed cons [ID]; mammogram is least often cancer screen for clinician to ask PT if want to be tested [COM]; mean decision processing scores are low across 3 cancer screens but lowest for mammogram [FD]

Women value grounding in real-world examples [ID], connection to medical research (\#s give credibility) [ID], and transparent enumeration of outcomes [ID]. Incorporating comprehension checks into DA will help identify misapprehension of graphically presented data and correct misunderstanding [ID]

Describes decision aid content (Box 2) [ID]

DA increased numerical and concept knowledge, decreased undecided, increased intended to decline mamm (same rate intended to mamm)

$1 / 2$ felt no bias, more thought biased against mamm than for mamm; most women recommend decision aid

Impact of this approach not formally evaluated. Offers decision-making model:

1. Discussion of PT's role in decision-making [FD]

2. Discussion of clinical issue and nature of decision to make [FD]

3. Discussion of alternatives [ID]

4. Discussion of benefits and risks of each alternative [ID]

5. Discussion of uncertainties [ID]

6. Assess PT's understanding [COM]

7. Exploration of PT's preferences [COM]

Describes process of developing decision aid its content [ID]; positive pilot feedback from women and clinicians.

Adherence improved with simply talking about it [COM], enthusiasm [COM], explanation, [ID] elicitation of barriers [COM], responsiveness to PT concerns [COM]; quality and content of recommendation may have additional and important bearing on PT's decision to get screened (strong predictor of adherence was amount of clinician enthusiasm and encouragement perceived by pts) [COM] 
Table 2. (continued)

\begin{tabular}{|c|c|}
\hline $\begin{array}{l}\text { First author/year/ } \\
\text { country }\end{array}$ & $\begin{array}{l}\text { a. Subjects } \\
\text { b. Setting } \\
\text { c. Methodology } \\
\text { d. Intervention (if applicable) }\end{array}$ \\
\hline $\begin{array}{l}\text { Petrova } 2015 \\
\text { USA }^{32}\end{array}$ & $\begin{array}{l}\text { a. Women/mammogram } \\
\text { b. Community sample } \\
\text { c. RCT comparing formats for numerical } \\
\text { information }\end{array}$ \\
\hline $\begin{array}{l}\text { Scariati } 2015 \\
\text { USA }^{28}\end{array}$ & $\begin{array}{l}\text { a. Women/mammogram } \\
\text { b. Social media sampling } \\
\text { c. Pre-post pilot test o } \\
\text { d. Web-based mammography DA for women to } \\
\text { use independently }\end{array}$ \\
\hline $\begin{array}{l}\text { Schonberg } 2014 \\
\text { USA }^{27}\end{array}$ & $\begin{array}{l}\text { a. Women }(75-89 \mathrm{y}) / \text { mammography } \\
\text { b. Clinic setting } \\
\text { c. Pre-post trial } \\
\text { d. Mammography DA pamphlet for women } 75 \text { - }\end{array}$ \\
\hline Seitz 2016 USA $^{33}$ & $\begin{array}{l}\text { a. Women }(35-49 \mathrm{y}) / \text { mammography } \\
\text { b. Community sample } \\
\text { c. RCT } 2 \text { (high vs low BCa risk) } \times 8 \text { (informatio } \\
\text { presentation) factorial design }\end{array}$ \\
\hline $\begin{array}{l}\text { Spring } 2016 \\
\text { USA }^{34}\end{array}$ & $\begin{array}{l}\text { a. Women }(40+y) / \text { mammography } \\
\text { b. National sample (HINTS) } \\
\text { c. Cross-sectional survey }\end{array}$ \\
\hline $\begin{array}{l}\text { Tisnado } 2015 \\
\text { USA }^{35}\end{array}$ & $\begin{array}{l}\text { a. Clinicians } \\
\text { b. Clinic setting } \\
\text { c. Pilot qualitative feedback w/ focus group } \\
\text { d. DA for geriatricians }\end{array}$ \\
\hline $\begin{array}{l}\text { Torres } 2013 \\
\text { USA }^{36}\end{array}$ & $\begin{array}{l}\text { a. Women (Latina)/mammogram } \\
\text { b. Community sample } \\
\text { c. Qualitative interview }\end{array}$ \\
\hline
\end{tabular}

\section{SDM elements [category designation]}

Visual aids increase comprehension among pts. who perceived breast cancer as moderately severe compared to text or fact box formats [ID]; increased comprehension is related to less strong intent to participate in screening and increased desire for SDM

Describes decision aid content (Box 2) [ID]

Use of decision aid reduced decision conflict $\mathrm{w} /$ no change in mamm intention; identified values women endorsed most: catch BCa early, peace of mind; other high and moderate values: avoid false negative, avoid false positive, overdiagnosis, radiation exposure [FD]

Describes decision aid content (Box 2) [ID]

Decision aid use increased knowledge of risks/benefits; decreased intent to screen; increased discussion regarding mamm as documented in medical record; non-sig decrease in decision conflict

For low-risk women, all risk-based intervention conditions increased accuracy of perceived BCa risk and intent to wait until age 50 to start mamm; inclusion of exemplars increased accuracy of personal risk [ID] For high-risk women, only brief intervention with untailored exemplars [ID] increased intention to screen at 40 , but no condition decreased intention

Between 2011 and 2014, less than half women received communication regarding mamm choice from clinician [ID]

Clinician communication that there is a choice increased mamm screen in 40-49 y women [ID]

Black women $34 \%$ less likely report clinician communication about choice [ID] compared to white women

Clinician criteria for decision-making: life expectancy, cognitive function, physical function, comorbidities, QOL, PT interest in mamm, treatment, family $\mathrm{h} / \mathrm{o} \mathrm{BCa}[\mathrm{FD}]$

Influential factors for mamm include: education about rights as PT and empowered to ask questions of clinicians [FD]; few mention clinician recommendation as reason [COM]. Communication w/ clinician challenged by language barriers [FD].; prefer information from MD vs RN, but RN may be easier to talk to because often a woman and have more time but are also perceived as less knowledgeable [COM]

Authors suggest: promote more communication and assertiveness with clinician $[\mathrm{FD}]$, empower women with confidence and resources to ask questions [FD]; increase availability of cultural and language appropriate print materials at community level [ID]; recognize and address local communication and geographic variability to access, screening adherence, health system issues [FD]

$\begin{array}{ll}\text { Wu } 2013 \text { USA }^{37} & \begin{array}{l}\text { a. Heterogeneous screening } \\ \text { b. Clinic setting } \\ \text { c. Controlled hybrid type 2 } \\ \text { implementation-effectiveness trial } \\ \text { d. MeTree (family health history risk assessment } \\ \text { tool) }\end{array} \\ \text { Zickmund 2010 } & \begin{array}{l}\text { a. Heterogeneous screening (breast, prostate, and } \\ \text { colon cancers) } \\ \text { USA }\end{array} \\ & \begin{array}{l}\text { b. National sample (DECISIONS Survey) } \\ \text { c. Cross-sectional survey }\end{array}\end{array}$

Mamm discussion occurred $59 \%$ of visits, more common of older age [ID]

BCa screen least frequent decision where PTs are asked preferences $(40 \%)$ [COM]; BCa least likely to report discussion of pros/cons (20\%) [ID]; in general, discussion of cons [ID] decreased decision confidence (but maybe false confidence without a balanced discussion)

ID information delivery and patient education, COM interpersonal clinician-patient communication, FD framework for the decision, BCa breast cancer, $P T$ patient, MD physician, RN nurse, DM decision-making, Mamm mammogram, y years old

associated with decreased confidence in the patient's decision; however, this may be a "false confidence" without a balanced discussion. ${ }^{38}$ Regardless of format, materials and communication need to be culturally and language appropriate to facilitate understanding. ${ }^{37}$ Forbes identified a particular challenge in meeting women's needs to keep information simple and to resist the call from experts for detail and caveats that convey scientific precision. ${ }^{18}$

Personalized risk estimates incorporated within communication interventions can enhance informed choice. ${ }^{20}$ Systematic reviews have examined the extent of literature and have identified preferred formats to deliver complex risk information. Specifically, absolute risk reduction (ARR) as compared to relative risk reduction is more understandable to both patients and clinicians. ${ }^{15}$ Natural frequency is superior to percentage or probability for ease of understanding and interpretation, ${ }^{15,17,27,30}$ and women prefer clear enumeration of outcomes. ${ }^{25}$ Additionally, in an RCT comparing formats of presenting numerical information, women who perceived the consequences of breast cancer as most serious, visual aids increase comprehension in comparison to text or fact box formats. ${ }^{32}$ Pasternack ${ }^{30}$ argues that all textual modalities should be used, including numbers, text, and visual images, 
to promote understanding. Yet Forbes ${ }^{18}$ received feedback that for a leaflet designed to provide balanced information about cancer screening benefits and harms, the women found numbers overwhelming and the infographics were also unhelpful. Sertz demonstrated in a RCT that the use of typical models or exemplars has been shown to increase accuracy of personal risk for breast cancer. ${ }^{33}$ Women value grounding risk information into real-world examples and the connection of information to medical research for credibility. ${ }^{25}$

Research has also identified specific language that is more favorable for discussing breast cancer risk factors. Overall tone should be neutral and balanced, ${ }^{27,30}$ using a personal and active voice (e.g., "you" rather than "one"). ${ }^{30}$ Women prefer the term "overtreatment" to "overdiagnosis" 18 and yet Forbes concludes that overall it is best to avoid either term in favor of descriptions such as "cancers that would otherwise never have been found or caused harm" or "never become lifethreatening." Women in this study also preferred the term "lives saved" in context of mortality benefit. ${ }^{18}$ Pasternack identified the use of "positive" and "negative" in the context of test results are problematic terms and should be replaced with lay descriptive terms such as "normal test result" rather than "negative. ${ }^{30}$ Positive framing (e.g., "9 of 10 lives saved") or both positive and negative framing (e.g., "1 of 10 lives lost") is preferred over negative framing alone. ${ }^{27,30}$

A limited number of studies included a decision aid intervention with evidence of benefit in decision-making. ${ }^{18,26-28}$ These decision aids where structured around multiple content areas. Box 2 lists the content areas found within these decision aids. Mathieu ${ }^{26}$ tested their online mammography decision aid in a RCT compared to a delayed access control group. The decision aid contained general information regarding the screening decision, likely outcomes of both screening and not screening, and value clarification, and was positively reviewed by participants. Findings indicate that the use of the decision aid increased numerical and concept knowledge and decreased indecision regarding mammography, but increased the number of women who intended to decline mammography.

Interpersonal Clinician-Patient Communication. Characteristics about the patient-clinician relationship and interpersonal

\section{Box 2 Elements of decision aid content}

\begin{tabular}{l}
\hline \hline Introduction \\
Purpose and intended audience \\
General information and explains there is a decision to make ${ }^{26}$ \\
Define screening and explored general population risks \\
Information on modifiable and non-modifiable breast cancer risk \\
factors 27,28 \\
Likely outcomes of screening and not screening and chances of each of \\
these outcomes occurring \\
Mammogram Information, e.g., screening process, safety, false positives \\
or negatives $^{28}$ \\
Breast cancer treatment information \\
Values clarification \\
Summary
\end{tabular}

interaction are included in the COM category. Eight articles addressed a key element in this category.

Joint communication between the patient and clinician is essential to SDM. A patient's adherence to mammography recommendations was found to improve with simply talking about mammography with the clinician, explanation, elicitation of barriers, and responsiveness to patients' concerns, while the patient's perception of the clinician's enthusiasm and encouragement for mammography were particularly strong predictors. ${ }^{31}$ Shared decision-making models suggest clinicians need to assess the woman's understanding of the information presented and explore her personal preferences. ${ }^{29}$ Yet, among cancer screening (e.g., breast, prostate, and colon cancer screens), clinicians were least likely to illicit patient input in the decision-making process, ${ }^{22}$ solicit patient preferences, ${ }^{38}$ or ask if the patient wants to be tested for breast cancer. $^{24}$

Framework of the Decision. Breast cancer screening SDM takes place within a broader framework of personal beliefs, values and understandings about mammography and health care decision-making. Nine of the articles addressed a key element in the FD category.

SDM requires understanding that there is a choice to be made, ${ }^{29}$ and perceived choice for mammography positively correlates with screening. ${ }^{23,34}$ Yet, among breast, prostate, colon cancer screenings, women considering mammography were least likely to believe they had a choice, ${ }^{17}$ and overall mean decision processing scores are low for cancer screening, but lowest for mammography. ${ }^{24}$ Furthermore, fewer African American than Caucasian women report clinician communication about having a choice. ${ }^{34}$

Perceived increased risk for breast cancer is associated with increased information seeking alone and with clinicians. ${ }^{19}$ However, women differ in the level of confidence to take an active role in decision-making. Specifically, African American women express a need to be prepared for a discussion with their clinician whereas Latina women are more likely to defer to the clinician's recommendation. ${ }^{16}$ This suggests a need to promote women to have more communication and assertiveness with their clinicians and empower women with confidence and resources to ask questions. ${ }^{37}$

Best practices in SDM need to consider multiple cultural perspectives. For example, the notion of weighing benefits to harms seemed irrelevant to many African American and Latina women. ${ }^{16}$ Some African American and Latina women expressed skepticism for information regarding risks of mammography; others thought this information had merit, however, were concerned for their own ability to sift through inconsistent information and act against their doctor's recommendation. ${ }^{16}$

Cultural frameworks directly influence clinician-patient communication. At a basic level, language barriers challenge communication with clinicians. ${ }^{36}$ More complex and less easily perceived barriers to communication include perceptions of the clinician's expertise and mistrust or misunderstanding of 
the clinician, information, and/or medical system. Without significant trust-building efforts, it is difficult to predict whether increased involvement with their clinician would necessarily influence appropriate use of mammography. ${ }^{16}$ Competing interests exist in women's preference for information from their clinician, yet they may be more comfortable and thus more likely to talk with a nurse because the nurse is often a woman and tends to have more time with the patient; however, Latina women actually perceived the nurses as less knowledgeable. $^{36}$

Furthermore, women and clinicians bring personal values to the decision-making process. The values women endorsed most were catching breast cancer early and peace of mind; other high to moderately important values include avoiding false negatives, avoiding false positives, overtreatment, and limiting radiation exposure. ${ }^{27,28}$ Clinicians might consider different values or risk factors than the women do. A focus group of geriatricians identified the criteria they considered important for the basis of breast cancer screening decisionmaking in elderly women, which included the woman's life expectancy, cognitive function, physical function, comorbidities, quality of life, interest in mammography, treatment, and family history of breast cancer. ${ }^{35}$

\section{DISCUSSION}

Current literature elucidates a number of key elements for supporting women and their clinicians in breast cancer screening SDM. These elements are particularly important for women ages 40-50 when routine mammography is more controversial and guidelines recommend SDM for personalized care. $^{3,5}$

Articles addressing mammography information delivery and patient education identified specific content and formats for content presentation. Research highlights the importance of educating women about both the benefits and the risks of mammography, ${ }^{18,21,29}$ the latter of which has been particularly absent in mammography education. ${ }^{22,24,38}$ Formats such as absolute risk reduction, natural frequencies, constant denominators, descriptive, everyday language rather than technical jargon, visual aid, and real-world examples are preferred by women, benefit comprehension, and facilitate SDM. ${ }^{15,17,20,25,27,30}$

The interpersonal communication between a woman and her clinician is a critical mechanism for SDM deliberation. Important roles for clinicians include assessing the woman's information understanding and exploring personal preferences. ${ }^{29}$ The clinician's demeanor, including enthusiasm, encouragement, and responsiveness to concerns, can facilitate effective SDM. ${ }^{31}$ Interpersonal factors related to the patientclinician relationship or communication exchange highlight the social context of SDM.

SDM occurs within a framework of modifiable and unmodifiable influences, both within and beyond the context of breast cancer screening. The basic framework for the decision relies on the woman understanding that she has a choice whether to have a mammogram and that there are factors to consider in making that choice. ${ }^{23,29,34}$ Beyond education and communication in SDM, many sociocultural factors impact SDM, including race, ethnicity, trust of the medical system, self-efficacy to engage in decision-making, and perceived risk for breast cancer. ${ }^{16,34,36,38}$ These factors work to influence a woman's engagement with information and processes of education, as well as the quality and value of interpersonal communication between the woman and her clinician.

While many of the key elements identified address features of existing SDM models, ${ }^{8-11}$ our findings speak beyond the elements of the deliberation to highlight the importance of personal patient, clinician, and sociocultural factors that influence breast cancer screening SDM. This relationship is illustrated in Fig. 3. A woman's individual characteristics (i.e., race, culture, language fluency, trust of and self-efficacy in medical system, and values) influence her willingness and ability to seek mammography information, participate in discussion, and make and adhere to decisions. Clinician characteristics have a similar impact on engagement and facilitation of SDM and include sex, competence in risk communication and SDM, biases toward (or against) breast cancer screening, beliefs about patient values, personal values, enthusiasm, and time constraints. Many clinicians find mammography guidelines confusing and are minimally aware of a number of personal breast cancer risk factors to facilitate ideal breast cancer screening SDM. ${ }^{12}$ The sociocultural context influences both the individuals involved and the personal and professional setting in which the clinical interaction for SDM is taking place. Therefore, consideration of these broader key elements influencing the breast cancer screening SDM process are necessary for teaching and guiding clinicians and developing culturally sensitive supportive materials and decision aids to support them and their patients.

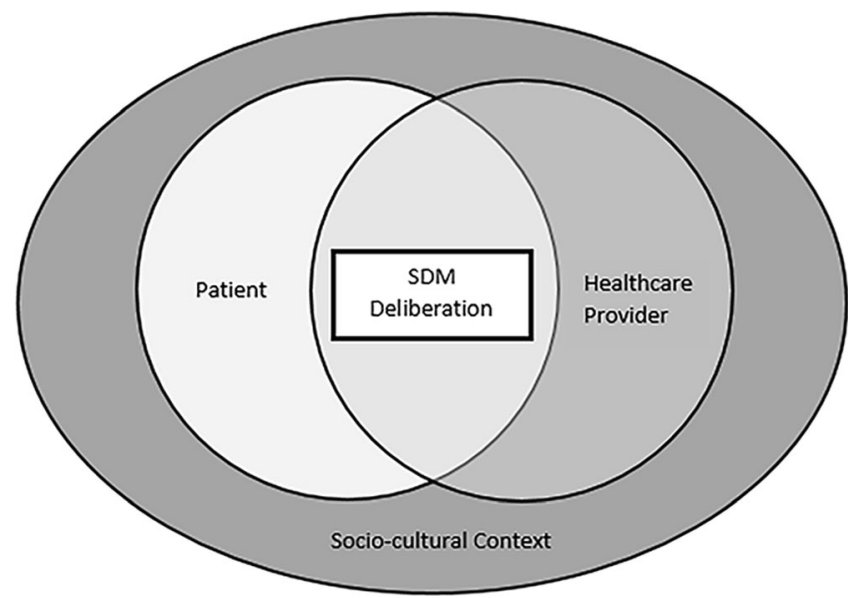

Figure 3 Relationship between key elements of shared decisionmaking. 


\section{Limitations}

Quality of Evidence. Our search yielded 24 articles for inclusion, with heterogeneity of aims, methods, and outcomes, with few intervention studies. Accordingly, metaanalysis was not possible for identifying the effects of a variety of SDM elements that were extracted from the articles. This prevented evaluation of which elements have the strongest support, or prioritizing more critical versus less critical elements of breast cancer screening SDM. Furthermore, aside from the literature on expression of risk rations, there is limited research that examines specific individual elements of shared decision-making for their unique merits within breast cancer screening decision-making.

Potential Biases in Review Process. Recent recommendations for breast cancer screening SDM are in the context of controversy for annual screening in women ages 4050 years. However, our literature selection criteria included all ages of women. While this may limit the specificity of findings for women age 40-50 years, we expanded our inclusion for this initial review to increase the opportunity for finding key elements, as literature specific to the younger age group would have been limited.

Furthermore, we narrowed the scope of our review to articles specifically within the context of mammography given the goals of our broader study. It is noteworthy that a vast amount of research exists on SDM, in a variety of health contexts. Yet inclusion of all elements of SDM was beyond the scope and feasibility of our study. Narrowing the scope afforded more specific findings to the nature of mammography and breast cancer risk considerations and nuance to this particular decision-making context. However, there is the risk that this review did not identify other applicable key elements that are present in broader studies.

Due to limited resources, we did not search Embase or the Cochrane Central Registry of Controlled Trials. We also restricted the selection of articles to those published in English. These and potentially other databases, along with non-English article, may have yielded additional evidence not reported here.

Search criteria included articles published in 2009 or later. In needing to set a cut-off date, this year offered a reasonable rationale given 2008 brought about changes in mammography guidelines and initial recommendations for SDM. Therefore, we searched for articles that would potentially address mammography within the cultural context of existing guidelines and emphasis on SDM. Accordingly, there may be older literature that could potentially hold relevant evidence for key elements, but that was beyond the scope of this review.

\section{Practice Implications}

This scoping review of the literature provides important information for clinicians counseling women in age ranges for which guidelines differ about breast cancer screening. Box 1 provides an overview highlighting elements that are most relevant to clinical practice. Expanding on the basic tenets of SDM, our findings underscore the importance of the relationship between the patient and clinician and the necessity of spelling out each step in the SDM process. The clinician needs to be explicit in telling a woman that she has a choice about whether to get a mammogram and the benefits and harms of screening mammography. Finally, clinicians need to be aware of sociocultural factors that can influence their relationships and their patients' decision-making processes and attempt to identify and address these factors.

Acknowledgements: Contributors: We would like to thank Elizabeth Jacobs, MD, for her work in reviewing articles and input in discussions of coding themes.

Corresponding Author: Lori L. DuBenske, PhD; School of Medicine and Public Health, University of Wisconsin-Madison, Madison, WI, USA (e-mail: dubenske@wisc.edu).

Funders Funding for this project was provided by the CommunityAcademic Partnerships core of the University of Wisconsin Institute for Clinical and Translational Research (UW ICTR), grant 9 U54 TR000021 from the National Center for Advancing Translational Sciences (previously grant 1 UL1 RRO25011 from the National Center for Research Resources), the University of Wisconsin Carbone Cancer Center, support grant P3O CAO1452O, and the NIH National Cancer Institute grant K24 CA194251. The content is solely the responsibility of the authors and does not necessarily represent the official views of the National Institutes of Health or other funders.

\section{Compliance with Ethical Standards:}

Conflict of Interest: Helene McDowell has received payment for consultancy services she has provided to HealthDecision Support Inc. All other authors declare no conflicts of interest.

\section{REFERENCES}

1. Centers for Disease Control and Prevention. Leading cause of death in females, 2014 (current listing). Available at: https://www.cdc.gov/women/lcod/2014/all-females/index.html. Accessed March 8, 2018.

2. American Cancer Society. Cancer facts and figures 2016. Available at: https://www.cancer.org/research/cancer-facts-statistics/all-cancerfacts-figures/cancer-facts-figures-2016.html. Accessed on March 8, 2018.

3. US Preventive Services Task Force. Breast cancer screening. Available from: https://www.uspreventiveservicestaskforce.org/Page/Document/ UpdateSummaryFinal/breast-cancer-screening 1 ?ds=1\&s=breast $\% 20$ cancer\%20screening. Accessed March 8, 2018.

4. Oeffinger KC, Fontham EH, Etzioni R, et al. Breast cancer screening for women at average risk: 2015 guideline update from the American Cancer Society. JAMA. 2015;314(15):1599-614.

5. American College of Obstetricians and Gynecologists. Breast cancer risk assessment and screening in average-risk women. Practice Bulletin No. 179. Obstet Gynecol 2017;130:e1-16.

6. Lee CH, Dershaw DD, Kopans D, Evans P, Monsees B, Monticciolo D, et al. Breast cancer screening with imaging: recommendations from the Society of Breast Imaging and the ACR on the use of mammography, breast MRI, breast ultrasound, and other technologies for the detection of clinically occult breast cancer. J Am Coll Radiol. 2010;7(1):18-27

7. Kerlikowske K. Progress toward consensus on breast cancer screening guidelines and reducing screening harms. JAMA Intern Med. 2015;20:1-

8. Sheridan SL, Harris RP, Woolf SH. Shared decision making about screening and chemoprevention: a suggested approach from the US Preventive Services Task Force. Am J Prev Med. 2004;26(1):56-66. 
9. Agency for Healthcare Research and Quality. The SHARE approach. Available at: https://www.ahrq.gov/professionals/education/curriculum-tools/shareddecisionmaking/index.html. Accessed March 8, 2018

10. Price D. Sharing clinical decisions by discussing evidence with patients. Perm J. 2005;9(2):70-73.

11. Elwyn G, Frosch D, Thomson R, Joseph-Williams N, Lloyd A Kinnersley P, Cording E, Tomson D, Dodd C, Rollnick S, Edwards A. Shared decision making: a model for clinical practice. J Gen Intern Med. 2012;27(10):1361-7.

12. DuBenske LL, Schrager S, McDowell H, Wilke LG, Trentham-Dietz A Burnside ES. Mammography screening: gaps in patient's and physician's needs for shared decision-making. Breast J. 2017;23(2):210-4.

13. Liberati A, Altman DG, Tetzlaff $\mathbf{J}$, et al. The PRISMA statement for reporting systematic reviews and meta-analyses of studies that evaluate health care interventions: explanation and elaboration. PLoS Med. 2009;6(7).

14. Colquhoun HL, Levac D, O'Brien KK, Straus S, Tricco AC, Perrier L, Kastner M, Moher D. Scoping reviews: time for clarity in definition, methods, and reporting. J Clin Epidemiol. 2014;67(12):1291-4.

15. Ahmed H, Naik G, Willoughby H, Edwards AG. Communicating risk. BMJ. 2012;344.

16. Allen JD, Bluethmann SM, Sheets M, Opdyke KM, Gates-Ferris K, Hurlbert M, Harden E. Women's responses to changes in US Preventive Task Force's mammography screening guidelines: results of focus groups with ethnically diverse women. BMC Public Health. 2013;13(1):1169.

17. Barrett B, McKenna P. Communicating benefits and risks of screening for prostate, colon, and breast cancer. Fam Med Kansas City. 2011;43(4):248

18. Berlin L. Point: mammography, breast cancer, and overdiagnosis: the truth versus the whole truth versus nothing but the truth. J Am Coll Radio. 2014;11(7):642-7

19. Dillard AJ, Couper MP, Zikmund-Fisher BJ. Perceived risk of cancer and patient reports of participation in decisions about screening: the DECISIONS study. Med Decis Making. 2010;30(5_suppl):96-105.

20. Edwards AG, Naik G, Ahmed H, Elwyn GJ, Pickles T, Hood K, Playle R. Personalised risk communication for informed decision making about taking screening tests. Cochrane Libr. 2013.

21. Forbes LJ, Ramirez AJ, Expert group on Information about Breast Screening. Offering informed choice about breast screening. J Med Screen. 2014;21(4):194-200.

22. Fowler FJ, Gerstein BS, Barry MJ. How patient centered are medical decisions?: Results of a national survey. JAMA Intern Med. 2013;173(13): 1215-21.

23. Gunn CM, Soley-Bori M, Battaglia TA, Cabral H, Kazis L. Shared decision making and the use of screening mammography in women younger than 50 years of age. J Health Commun. 2015;20(9):1060-6.

24. Hoffman RM, Elmore JG, Fairfield KM, Gerstein BS, Levin CA, Pignone MP. Lack of shared decision making in cancer screening discussions. Amer J Prev Med. 2014;47(3):251-9.
25. Klein KA, Watson L, Ash JS, Eden KB. Evaluation of risk communication in a mammography patient decision aid. Patient Educ Couns. 2016;99(7): 1240-8.

26. Mathieu E, Barratt AL, McGeechan K, Davey HM, Howard $\mathbf{K}$, Houssami N. Helping women make choices about mammography screening: an online randomized trial of a decision aid for 40-year-old women. Patient Educ Couns. 2010;81(1):63-72.

27. Schonberg MA, Hamel MB, Davis RB, Griggs MC, Wee CC, Fagerlin A, Marcantonio ER. Development and evaluation of a decision aid on mammography screening for women 75 years and older. JAMA Inter Med. 2014;174(3):417-24.

28. Scariati P, Nelson L, Watson L, Bedrick S, Eden KB. Impact of a decision aid on reducing uncertainty: pilot study of women in their 40s and screening mammography. BMC Med Inform Decis Mak. 2015;15(1):89.

29. Nekhlyudov L, Braddock III CH. An approach to enhance communication about screening mammography in primary care. J Women's Health. 2009; 18(9): 1403-12.

30. Pasternack I, Saalasti-Koskinen U, Mäkelä M. Decision aid for women considering breast cancer screening. Int $J$ Technol Assess Health Care. 2011;27(4):357-62.

31. Peterson EB, Ostroff JS, DuHamel KN, D'Agostino TA, Hernandez M, Canzona MR, Bylund CL. Impact of provider-patient communication on cancer screening adherence: a systematic review. Prev Med. 2016;93:96105.

32. Petrova D, Garcia-Retamero R, Cokely ET. Understanding the harms and benefits of cancer screening: a model of factors that shape informed decision making. Med Decis Making. 2015;35(7):847-58.

33. Seitz HH, Gibson L, Skubisz C, Forquer H, Mello S, Schapira MM, Armstrong K, Cappella JN. Effects of a risk-based online mammography intervention on accuracy of perceived risk and mammography intentions. Patient Educ Couns. 2016;99(10):1647-56.

34. Spring LM, Marshall MR, Warner ET. Mammography decision making: trends and predictors of provider communication in the Health Information National Trends Survey, 2011 to 2014. Cancer. 2017;123(3):401-9.

35. Tisnado DM, Moore AA, Levin JR, Rosen S. Developing and testing a decision aid for use by providers in making recommendations: about mammography screening in older women. J App Gerontol. 2015;34(3):343-58.

36. Torres E, Erwin Do, Trevino M, Jandorf L. Understanding factors influencing Latina women's screening behavior: a qualitative approach. Health Educ Res. 2012;28(5):772-83.

37. Wu RR, Orlando LA, Himmel TL, et al. Patient and primary care provider experience using a family health history collection, risk stratification, and clinical decision support tool: a type 2 hybrid controlled implementation-effectiveness trial. BMC Fam Pract. 2013;14(1):111.

38. Zikmund-Fisher BJ, Couper MP, Singer E, et al. Deficits and variations in patients' experience with making 9 common medical decisions: the DECISIONS survey. Med Decis Making. 2010;30(5_suppl):85-95. 